\title{
HIGHER ORDER SUPERSYMMETRIES AND FERMIONIC CONSERVATION LAWS OF THE SUPERSYMMETRIC EXTENSION OF THE KdV EQUATION
}

\author{
Paul H.M. KERSTEN \\ Faculty of Applied Mathematics, University of Twente, P.O. Box 217, 7500 AE Enschede, The Netherlands
}

Received 26 May 1988; revised manuscript received 15 September 1988; accepted for publication 30 September 1988 Communicated by A.P. Fordy

\begin{abstract}
By the introduction of nonlocal bosonic and fermionic variables we construct a recursion symmetry of the super KdV equation, leading to a hierarchy of bosonic symmetries and one of fermionic symmetries. The hierarchies of bosonic and fermionic conservation laws arise in a natural way in the construction.
\end{abstract}

\section{Introduction}

In two recent papers [1,2] we studied symmetries and nonlocal symmetries of the super $\mathrm{KdV}$ and super $m K d V$ equation [3], and we derived a nonlocal higher order symmetry leading to the recursion operator for higher order symmetries in a straightforward way, not relying on the bi-hamiltonian structure of the equations. All symmetries discussed there were even [4] or bosonic symmetries. These equations however, are not supersymmetric in the sense that they admit an odd [4] or fermionic symmetry. Often fermionic or odd symmetries are called supersymmetries. However, there is known a supersymmetric extension of the KdV equation by Manin and Radul [5] which admits a supersymmetry. This supersymmetric extension admits an infinite hierarchy of even higher order or generalised symmetries and conserved quantities. No recursion operator for symmetries is known in this case [6]. Moreover nothing is known about the existence of fermionic conservation laws besides the only one arising from the supersymmetry.

In this paper we shall construct a nonlocal symmetry of the supersymmetric $\mathrm{KdV}$ equation, which together with the already known supersymmetry generates a graded Lie algebra of symmetries, comprising a hierarchy of bosonic higher order symmetries and a hierarchy of nonlocal higher order supersymmetries. The familiar supersymmetry is just the first member of the hierarchy. Moreover, higher order even and odd conserved quantities arise in a natural and elegant way in the construction of the infinite dimensional graded Lie algebra. The construction of higher order even symmetries is given in section 2, while the construction of the above mentioned nonlocal symmetry together with the graded Lie algebra structure is given in section 3.

\section{Higher order symmetries of the supersymmetric $\mathrm{KdV}$ equation}

The existence of higher order even [4] symmetries of the supersymmetric extension of the $\mathrm{KdV}$ equation

$u_{t}=-u_{x x x}+6 u u_{x}$

shall be discussed. We start at the supersymmetric extension given by Mathieu [6], i.e.

$u_{t}=-u_{3}+6 u u_{1}-a \varphi \varphi_{2}$,

$\varphi_{t}=-\varphi_{3}+(6-a) \varphi_{1} u+a \varphi u_{1}$.

In (2.2) integer indices refer to differentiation with respect to $x$, i.e. $u_{3}=u_{x x x} ; x, t, u$ are even, while $\varphi$ is odd [4]; the parameter $a$ is real. Taking $\varphi \equiv 0$ we get (2.1). Classical higher order symmetries are defined on the infinite jet bundle $\mathrm{J}^{\infty}(x, t ; u, \varphi)$ [7] and satisfy the symmetry condition 
$\mathscr{L}_{V}\left(\mathrm{D}^{\infty} \mathrm{I}\right) \subset \mathrm{D}^{\infty} \mathrm{I}$,

where $D^{\infty} I$ is the infinite prolongation of the exterior differential system I, describing the partial differential equation, by means of the action of the total partial derivative vector fields $\mathrm{D}_{x}, \mathrm{D}_{t}$ defined by

$\mathrm{D}_{x}=\partial_{x}+u_{x} \partial_{u}+\varphi_{x} \partial_{\varphi}+\ldots$,

$\mathrm{D}_{t}=\partial_{t}+u_{t} \partial_{u}+\varphi_{t} \partial_{\varphi}+\ldots$.

In (2.3) $\mathscr{L}_{V}$ denotes the Lie derivative with respect to the vector field $V$. Due to the fact that (2.4) satisfies (2.3) in an obvious way, the search for higher order symmetries (generalised symmetries) can be restricted to vertical vector fields; i.e. the components $\partial_{x}, \partial_{t}$ are taken to be zero.

The vertical vector fields are proved to have the following representation,

$V=f \partial_{u}+g \partial_{\varphi}+\left(\mathrm{D}_{x} f\right) \partial_{u_{1}}+\left(\mathrm{D}_{x} g\right) \partial_{\varphi_{1}}+\ldots$,

so we are only interested in the defining function $f, g$ of the vector field. The functions $f$ and $g$ are assumed to depend on a finite number of independent variables of the infinite jet bundle. In the graded case [4] at hand (2.2) we proceed in a similar way, keeping in mind the left module structure of vector fields. We restrict our search for higher order symmetries at this moment to even vector fields; moreover our search is for vector fields $V$ whose defining functions $f$ and $g(2.5)$ depend on $x, t, u, \varphi, u_{1}, \varphi_{1}, \ldots, u_{5}, \varphi_{5}$, the other components being obtained by prolongation (2.4), (2.5). More specifically

$f=f_{1}+f_{2} \varphi \varphi_{1}+f_{3} \varphi \varphi_{2}+f_{4} \varphi \varphi_{3}+f_{5} \varphi \varphi_{4}+f_{6} \varphi_{1} \varphi_{2}+f_{7} \varphi_{1} \varphi_{3}$,

$g=g_{1} \varphi+g_{2} \varphi_{1}+g_{3} \varphi_{2}+g_{4} \varphi_{3}+g_{5} \varphi_{4}+g_{6} \varphi_{5}$,

whereas in (2.6) $f_{1}, \ldots, f_{7}, g_{1}, \ldots, g_{6}$ are dependent on the even variables $x, t, u, \ldots, u_{5}$. Formula (2.6) is motivated by the standard grading in the classical sense of (2.2),

$\operatorname{deg}(x)=-1, \quad \operatorname{deg}(u)=2$,

$\operatorname{deg}(t)=-3, \quad \operatorname{deg}(\varphi)=\frac{3}{2}$,

and the results obtained for other cases [1].

The vector field $V(2.5),(2.6)$ has to satisfy the symmetry condition (2.3) which is equivalent to

$\mathscr{L}_{V}\left(u_{t}+u_{3}-6 u u_{1}+a \varphi \varphi_{2}\right) \doteq 0$,
$\mathscr{L}_{V}\left(\varphi_{t}+\varphi_{3}-(6-a) \varphi_{1} u-a \varphi u_{1}\right) \doteq 0$,

where " $=0$ " should be understood as equal to zero on the submanifold of the infinite jet bundle $\mathrm{J}^{\infty}(x, t$; $u, \varphi)$ defined by (2.2) and its differential consequences.

Conditions (2.8) lead to an overdetermined system of partial differential equations for the functions $f_{1}, \ldots, f_{7}, g_{1}, \ldots, g_{6}$. In order to obtain this overdetermined system we made use of the graded differential geometry package described in ref. [8]. Solving this overdetermined system of partial differential equations we obtain the following result:

Theorem 2.1. If $a=3$ there are four vector fields satisfying (2.8) under conditions (2.6), i.e.

$$
\begin{aligned}
X_{1} & =u_{1} \partial_{u}+\varphi_{1} \partial_{\varphi}, \\
X_{3} & =\left(u_{3}-6 u_{1} u+3 \varphi \varphi_{2}\right) \partial_{u}+\left(\varphi_{3}-3 \varphi_{1} u-3 \varphi u_{1}\right) \partial_{\varphi}, \\
X_{5} & =-\left(u_{5}-10 u_{3} u-20 u_{2} u_{1}+30 u_{1} u^{2}+5 \varphi \varphi_{4}\right. \\
& \left.+5 \varphi_{1} \varphi_{3}-20 u \varphi \varphi_{2}-20 u_{1} \varphi \varphi_{1}\right) \partial_{u}-\left(\varphi_{5}-5 u \varphi_{3}\right. \\
& \left.-10 u_{1} \varphi_{2}-10 u_{2} \varphi_{1}+10 u^{2} \varphi_{1}+20 u_{1} u \varphi-5 u_{3} \varphi\right) \partial_{\varphi}, \\
V & =-3 t X_{3}+x X_{1}+2 u \partial_{u}+\frac{3}{2} \varphi \partial_{\varphi} .
\end{aligned}
$$

If $a \neq 3$ then $X_{5}$ is not a symmetry of (2.2).

Next, our search is for odd vector fields (2.5) satisfying (2.8); the assumption on the functions $f$ and $g$ is

$f=f_{1} \varphi+f_{2} \varphi_{1}+f_{3} \varphi_{2}+f_{4} \varphi_{3}+f_{5} \varphi_{4}+f_{6} \varphi_{5}+f_{7} \varphi_{6}$,

$$
\begin{aligned}
g= & g_{1}+g_{2} \varphi \varphi_{1}+g_{3} \varphi \varphi_{2}+g_{4} \varphi \varphi_{3}+g_{5} \varphi \varphi_{4}+g_{6} \varphi \varphi_{5} \\
& +g_{7} \varphi_{1} \varphi_{2}+g_{8} \varphi_{1} \varphi_{3}+g_{9} \varphi_{1} \varphi_{4}+g_{10} \varphi_{2} \varphi_{3},
\end{aligned}
$$

where $f_{1}, \ldots, f_{7}, g_{1}, \ldots, g_{10}$ are dependent on $x, t, u, \ldots$, $u_{5}$. Solving the overdetermined system of partial differential equations resulting from conditions (2.8), (2.10) leads to:

Theorem 2.2. There exists only one odd vector field satisfying (2.10) and the symmetry condition (2.8), i.e.

$Y_{1 / 2}=\varphi_{1} \partial_{u}+u \partial_{\varphi}$.

In order to obtain the Lenard recursion operator we did proceed in a way similar to that discussed in refs. $[1,2]$, but unfortunately we were not successful. We 
shall discuss recursion formulas for symmetries in the next section.

\section{Nonlocal symmetries and hierarchies of symmetries and conserved quantities}

By the introduction of nonlocal variables [9] we derive a nonlocal symmetry for the supersymmetric $\mathrm{KdV}$ equation $(a=3)$,

$u_{t}=-u_{3}+6 u_{1} u-3 \varphi \varphi_{2}$,

$\varphi_{t}=-\varphi_{3}+3 \varphi_{1} u+3 \varphi u_{1}$,

which together with the supersymmetry $Y_{1 / 2}$ generates two hierarchies of symmetries. The even and odd conserved quantities arise in a natural way.

We first start with the observation that

$q_{1 / 2}=\int_{-\infty}^{x} \varphi \mathrm{d} x$

is a potential of $(3.1)$, i.e.

$\left(q_{1 / 2}\right)_{x}=\varphi, \quad\left(q_{1 / 2}\right)_{t}=-\varphi_{2}+3 \varphi u$.

The quantity $Q_{1 / 2}$ defined by

$Q_{1 / 2}=\int_{-\infty}^{\infty} \varphi \mathrm{d} x$

is a conserved quantity of the supersymmetric $\mathrm{KdV}$ equation (2.2), (3.1). We now make the following observation:

Theorem 3.1. The nonlocal vector field $Z_{1}$, defined by

$Z_{1}=\left(q_{1 / 2} \varphi_{1}\right) \partial_{u}+\left(q_{1 / 2} u-\varphi_{1}\right) \partial_{\varphi}$

is a nonlocal symmetry of (3.1). Moreover there is no nonlocal symmetry, linear with respect to $q_{1 / 2}$ which satisfies (2.2) for $a \neq 3$.

The vector field $Z_{1}$ together with the vector field $Y_{1 / 2}$ plays a fundamental role in the graded algebra of symmetries of (3.1). From now on, for obvious reasons, we shall restrict ourselves to (3.1).

Note. All odd variables $\varphi_{0} \varphi_{1} \ldots q_{1 / 2}$ etc. are of degree $n / 2$ where $n$ is odd (2.7). The vector fields $Z_{1}$ and $Y_{1 / 2}$ are even and odd respectively.
We now want to compute the graded Lie algebra with $Z_{1}$ and $Y_{1 / 2}$ as seed elemems, so the graded Lie bracket has to be calculated. In order to do so we have to prolong the vector field $Y_{1 / 2}$ towards the nonlocal variable $q_{1 / 2}$, or by just writing $Y_{1 / 2}$ for this prolongation to calculate the component $\partial_{q_{1 / 2}}$. The coefficient $Y_{1 / 2}^{q_{1 / 2}}$ has to be such that the vector field $Y_{1 / 2}$ leaves invariant eqs. (3.2b), i.e. the Lie derivative of $(3.2 \mathrm{~b})$ with respect to $Y_{1 / 2}$ is zero; i.e.

$\mathrm{D}_{x}\left(Y_{1 / 2}^{q_{1 / 2}}\right)-u=0$,

$\mathrm{D}_{t}\left(Y_{1 / 2}^{q_{1} / 2}\right)+u_{2}-3 u^{2}+3 \varphi \varphi_{1}=0$.

By (3.5), (3.6) we are led in a natural and elegant way to the introduction of the even nonlocal variable $p_{1}$, defined by

$p_{1}=\int_{-\infty}^{x} u \mathrm{~d} x$,

satisfying

$\left(p_{1}\right)_{x}=u, \quad\left(p_{1}\right)_{t}=-u_{2}+3 u^{2}-3 \varphi \varphi_{1}$,

i.e. $p_{1}$ is a potential of the supersymmetric $\mathrm{KdV}$ equation ( 3.1$)$; the integrability conditions being satisfied; conserved quantity $P_{1}$. Now the vector field $Y_{1 / 2}$ is given by

$Y_{1 / 2}=\varphi_{1} \partial_{u}+u \partial_{\varphi}+p_{1} \partial_{q_{1 / 2}}+\ldots$.

Computation of the commutator of $Z_{1}$ and $Y_{1 / 2}$ leads to a new vector field $Y_{3 / 2}$, given by

$$
\begin{gathered}
Y_{3 / 2}=\left[Z_{1}, Y_{1 / 2}\right]=\left(2 q_{1 / 2} u_{1}-p_{1} \varphi_{1}+u \varphi-\varphi_{2}\right) \partial_{u} \\
+\left(2 q_{1 / 2} \varphi_{1}-p_{1} u+u_{1}\right) \partial_{\varphi}+\ldots
\end{gathered}
$$

The vector field $Y_{3 / 2}$ is a new nonlocal odd symmetry of (3.1). Note that, as a polynomial in $q_{1 / 2}$ and $p_{1}$ the coefficients in (3.9) are just the symmetries $2 X_{1}$ and $-Y_{1 / 2}$ respectively.

We now proceed by induction. In order to compute the graded Lie bracket $\left[Z_{1}, Y_{3 / 2}\right]$ we first have to compute the prolongation of $Z_{1}$ towards the nonlocal variables $p_{1}$ and $q_{1 / 2}$, i.e. the $\partial_{p_{1}}$ and $\partial_{q_{1 / 2}}$ components of the vector field, again denoted by $Z_{1}$. An easy calculation, the invariance of $(3.7 \mathrm{~b})$ by $Z_{1}$, shows that $Z_{1}^{p_{1}}$ has to satisfy

$\mathrm{D}_{x}\left(Z_{1}^{p_{1}}\right)=q_{1 / 2} \varphi_{1}$,

from which we have 
$Z_{1}^{p_{1}}=q_{1 / 2} \varphi$.

The $\partial_{q 1 / 2}$ component $Z^{q^{1 / 2}}$ however has to satisfy the invariance condition of (3.2b) by $Z_{1}$, i.e.

$\mathrm{D}_{x}\left(Z^{q^{1 / 2}}\right)-q_{1 / 2} u+\varphi_{1}=0$,

from which

$Z_{1}^{q_{1 / 2}}=q_{1 / 2} p_{1}-\varphi-\int_{-\infty}^{x} p_{1} \varphi \mathrm{d} x$.

So prolongation of $Z_{1}$ towards the nonlocal variable $q_{1 / 2}$ requires the formal introduction of the odd nonlocal variable $q_{3 / 2}$ defined by

$q_{3 / 2}=\int_{-\infty}^{x} p_{1} \varphi \mathrm{d} x$

where

$\left(q_{3 / 2}\right)_{x}=p_{1} \varphi$

$\left(q_{3 / 2}\right)_{t}=p_{1}\left(-\varphi_{2}+3 u \varphi\right)-u_{1} \varphi+u \varphi_{1}$,

the integrability condition on $(3.13 \mathrm{~b})$ being satisfied, which is easy to check; so $q_{3 / 2}$ is an odd nonlocal potential of (3.1), $Q_{3 / 2}$ being the associated odd and nonlocal conserved quantity. The vector field $Z_{1}$ is now given by

$$
\begin{aligned}
Z_{1} & =q_{1 / 2} \varphi_{1} \partial_{u}+\left(q_{1 / 2} u-\varphi_{1}\right) \partial_{\varphi}+\left(q_{1 / 2} p_{1}\right. \\
& \left.-\varphi-q_{3 / 2}\right) \partial_{q_{1 / 2}}+q_{1 / 2} \varphi \partial_{p_{1}} \\
& =q_{1 / 2} Y_{1 / 2}-\varphi_{1} \partial_{\varphi}-\left(\varphi+q_{3 / 2}\right) \partial_{q_{1 / 2}} .
\end{aligned}
$$

The prolongation of the vector field $Y_{3 / 2}$ towards the nonlocal variables $q_{1 / 2}, p_{1 / 2}$ are calculated from the invariance of $(3.2 b),(3.7 b)$ resulting in

$$
\begin{aligned}
& Y_{3 / 2}^{q_{1} / 2}=2 q_{1 / 2} \varphi-\frac{1}{2}\left(p_{1}\right)^{2}+u, \\
& Y_{1 / 2}^{p_{1}}=2 q_{1 / 2} u-p_{1} \varphi-\varphi_{1} .
\end{aligned}
$$

Computation of the graded Lie bracket $\left[Z_{1}, Y_{3 / 2}\right]$ leads to

$$
\begin{aligned}
& Y_{5 / 2}=\left[-2 q_{3 / 2} u_{1}+\frac{1}{2}\left(p_{1}\right)^{2} \varphi_{1}+p_{1}\left(\varphi_{2}-u \varphi\right)-4 u_{1} \varphi\right. \\
& \left.\quad-3 u \varphi_{1}+\varphi_{3}\right] \partial_{u}+\left[-2 q_{3 / 2} \varphi_{1}+\frac{1}{2}\left(p_{1}\right)^{2} u\right. \\
& \left.\quad+p_{1}\left(-u_{1}\right)+u_{2}-2 u^{2}+\varphi \varphi_{1}\right] \partial_{\varphi}+\left[-2 q_{3 / 2} u\right. \\
& \left.\quad+\frac{1}{2}\left(p_{1}\right)^{2} \varphi+p_{1} \varphi_{1}-4 u \varphi+\varphi_{2}\right] \partial_{p_{1}}+\left[-2 q_{3 / 2} p_{1} \varphi\right. \\
& \left.\quad+\frac{1}{8}\left(p_{1}\right)^{4}-\left(p_{1}\right)^{2} u+p_{1} u_{1}-\frac{1}{2} u^{2}-\varphi \varphi_{1}\right] \partial_{q 3 / 2},
\end{aligned}
$$

whereas the $\partial_{p_{1}}, \partial_{q_{3 / 2}}$ components of $Y_{5 / 2}$ are obtained by the invariance of $(3.7 b),(3.13 b)$. In order to obtain the prolongation of $Y_{5 / 2}$ towards the nonlocal variable $q_{1 / 2}$ we have to require the invariance of $(3.2 b)$, which results in the condition

$$
\begin{aligned}
& \mathrm{D}_{x}\left(Y_{\xi}^{q 1 / 2}\right)=-2 q_{3 / 2} \varphi_{1}+\frac{1}{2}\left(p_{1}\right)^{2} u-p_{1} u_{1}+u_{2} \\
& \quad-2 u^{2}+\varphi \varphi_{1},
\end{aligned}
$$

from which we have

$$
\begin{aligned}
& Y_{5}^{q_{1} / 2}=\frac{1}{6}\left(p_{1}\right)^{3}-p_{1} u+u_{1}-2 q_{3 / 2} \varphi \\
& +\int_{-\infty}^{x}\left[u^{2}+2\left(p_{1} \varphi\right) \varphi+\varphi \varphi_{1}-2 u^{2}\right] \mathrm{d} x \\
& \quad=\frac{1}{6}\left(p_{1}\right)^{3}-p_{1} u+u_{1}-2 q_{3 / 2} \varphi-\int_{-\infty}^{x}\left(u^{2}-\varphi \varphi_{1}\right) \mathrm{d} x .
\end{aligned}
$$

So expression (3.18) requires in an elegant way the introduction of $p_{3}$, defined by

$p_{3}=\int_{-\infty}^{x}\left(u^{2}-\varphi \varphi_{1}\right) \mathrm{d} x$,

where

$$
\begin{aligned}
& \left(p_{3}\right)_{x}=u^{2}-\varphi \varphi_{1}, \\
& \left(p_{3}\right)_{t}=4 u^{3}-2 u_{2} u+u_{1}^{2}-9 u \varphi \varphi_{1}+\varphi \varphi_{3}-2 \varphi_{1} \varphi_{2} .
\end{aligned}
$$

$p_{3}$ is a well-known potential, $P_{3}$ being the associated conserved quantity.

The commutator $Y_{7 / 2}=\left[Z_{1}, Y_{5 / 2}\right]$ requires the prolongation of the vector field $Z_{1}$ towards the nonlocal variable $q_{3 / 2}$, obtained by the invariance of (3.13b) by $Z_{1}$, so

$\mathrm{D}_{x}\left(Z^{q^{3 / 2}}\right)=Z_{1}\left(p_{1} \varphi\right)=\left(q_{1 / 2} \varphi\right) \varphi+p_{1}\left(q_{1 / 2} u-\varphi_{1}\right)$.

Integration of (3.20) leads to

$$
Z_{1}^{q_{3 / 2}}=\frac{1}{2}\left(p_{1}\right)^{2} q_{1 / 2}-p_{1} \varphi-\int^{\varkappa}\left[\frac{1}{2}\left(p_{1}\right)^{2} \varphi-u \varphi\right] d x .
$$

The new odd nonlocal variable $q_{s / 2}$ is formally defined by 
$q_{5 / 2}=\int_{-\infty}^{x}\left[\frac{1}{2}\left(p_{1}\right)^{2} \varphi-u \varphi\right] d x$

$q_{5 / 2}$ is a nonlocal odd potential of the supersymmetric KdV equation (3.1),

$$
\begin{aligned}
& \left(q_{5 / 2}\right)_{x}=\frac{1}{2}\left(p_{1}\right)^{2} \varphi-u \varphi, \\
& \left(q_{5 / 2}\right)_{t}=\frac{1}{2}\left(p_{1}\right)^{2}\left(-\varphi_{2}+3 u \varphi\right)+p_{1}\left(-u \varphi+u \varphi_{1}\right) \\
& \quad+u_{2} \varphi-u_{1} \varphi_{1}-4 u^{2} \varphi+u \varphi_{2} .
\end{aligned}
$$

Proceeding in this way, we obtain a hierarchy of nonlocal supersymmetries by induction,

$Y_{n+1 / 2}=\left[Z_{1}, Y_{n-1 / 2}\right], \quad n \in \mathbb{N}$.

The higher order even potentials $p_{1}, p_{3}, \ldots$ arise in a natural way in the prolongation of the vector fields $Y_{2 n+1 / 2}$ towards the nonlocal variable $q_{1 / 2}$, where the nonlocal superpotentials $q_{1 / 2}, q_{3 / 2}, q_{5 / 2}, \ldots$ are obtained in the prolongation of the recursion symmetry $Z_{1}$. A formal proof of these facts is beyond the scope of the presentation of these results and will be discussed elsewhere using the hamiltonian structure.

To obtain the graded Lie algebra structure of symmetries we calculate the graded Lie bracket of the vector fields derived so far. The result is remarkable and fascinating,

$$
\begin{aligned}
& {\left[Y_{1 / 2}, Y_{1 / 2}\right]=2 X_{1}, \quad\left[Y_{3 / 2}, Y_{3 / 2}\right]=2 X_{3},} \\
& {\left[Y_{5 / 2}, Y_{5 / 2}\right]=2 X_{5},}
\end{aligned}
$$

so the "squares" of the supersymmetries $Y_{1 / 2}, Y_{3 / 2}$, $Y_{5 / 2}$ are just the "classical" symmetries $2 X_{1}, 2 X_{3}, 2 X_{5}$ obtained in the previous section (2.9)! The other commutators are

$\left[Y_{1 / 2}, Y_{3 / 2}\right]=0, \quad\left[Y_{1 / 2}, Y_{5 / 2}\right]=-2 X_{3}$,

$\left[Y_{3 / 2}, Y_{5 / 2}\right]=0$,

while

$\left[X_{1}, X_{3}\right]=\left[X_{1}, X_{5}\right]=\left[X_{3}, X_{5}\right]=0$,

$\left[Z_{1}, X_{1}\right]=\left[Z_{1}, X_{3}\right]=\left[Z_{1}, X_{5}\right]=0$,

$\left[Y_{n+1 / 2}, X_{2 m+1}\right]=0, \quad n=0,1,2, \ldots ; m=0,1,2$.

The results are illustrated in fig. 1 . We conjecture that in this way we obtain an infinite hierarchy of nonlocal supersymmetries $\left(Y_{n+1 / 2} ; n \in \mathbb{N}\right)$ and an infinite

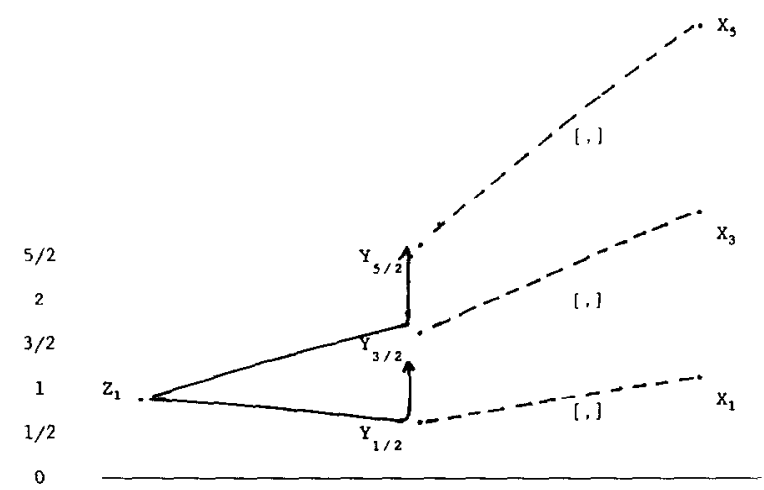

Fig. 1. The graded Lie algebra.

hierarchy of ordinary even higher order symmetries $\left(X_{2 n+1}, n \in \mathbb{N}\right)$, while the even and odd conserved quantities $P_{2 n+1}, Q_{n+1 / 2}(n \in \mathbb{N})$ are obtained by prolongation of the vector fields $Y_{n+1 / 2}$ and $Z_{1}$ respectively.

We finish this section with the following lemma on the graded Lie algebra structure:

Lemma 1. Let $X_{2 n+1}(n \in \mathbb{N})$ be defined by

$X_{2 n+1}=\frac{1}{2}\left[Y_{n+1 / 2}, Y_{n+1 / 2}\right]$

and assume

$\left[Z_{1}, X_{2 n+1}\right]=0, \quad n \in \mathbb{N}$,

then

$$
\begin{aligned}
& {\left[Y_{n+1 / 2}, Y_{m+1 / 2}\right]} \\
& =(-1)^{m-n} 2 X_{n+m+1}, \quad m-n \text { even, } \\
& =0, \quad m-n \text { odd, } \\
& {\left[Y_{n+1 / 2}, X_{2 m+1}\right]=0, \quad n, m \in \mathbb{N} \text {, }} \\
& {\left[X_{2 n+1}, X_{2 m+1}\right]=0, \quad n, m \in \mathbb{N} \text {. }}
\end{aligned}
$$

The proof of lemma 1 is straightforward [10].

\section{Conclusion}

By using the concept of nonlocal symmetries we obtain hierarchies of symmetries and supersymmetries for the supersymmetric KdV equation. The bosonic and fermionic conserved quantities arise in an elegant way. We took great advantage of the devel- 
oped software to handle graded differential geometry [8]

\section{References}

[1] P.H.M. Kersten and P.K.H. Gragert, J. Phys. A 21 (1988) L579.

[2] P.H.M. Kersten, Symmetries for the super modified KdV equation, Memorandum 691, University of Twente (1988).

[3] B.A. Kupershmidt, Phys. Lett. A 102 (1984) 213.

[4] B. Kostant, Lecture notes in mathematics, Vol. 570 (Springer, Berlin, 1977) pp. 177-306.

[5] Yu.I. Manin and A.O. Radul, Commun. Math. Phys. 98 (1985) 65 .
[6] P. Mathieu, Supersymmetric extension of the Korteweg-de Vries equation, Département de Physique, Université Laval, Quebcc (1987).

[7] F.A.E. Pirani, D.C. Robinson and W.F. Shadwick, Local jet bundle formulation of Bäcklund transformations, Mathematical physics studies 1 (Reidel, Dordrecht, 1979).

[8] P.K.H. Gragert and P.H.M. Kersten, Graded differential geometry in REDUCE 3, Memorandum 680, University of Twente (1988).

[9] I.S. Krasilshchik and A.M. Vinogradov, Acta Appl. Math. 2 (1984) 79.

[10] P.H.M. Kersten, Higher order supersymmetries and fermionic conservation laws of the supersymmetric extension of the $\mathrm{KdV}$ and $\mathrm{mKdV}$ equation, Memorandum 712, University of Twente (1988). 